\title{
CRIOTERAPIA: MÉTODOS E APLICACCÕES EM PESQUISAS BRASILEIRAS UMA REVISÃO SISTEMÁTICA
}

\section{Natamia Angeoles Lima}

Fisioterapeuta da Prefeitura Municipal de Coari (AM), Brasil.

\section{Vanderlane de Souza Duarte}

Docente Especialista do Instituto de Saúde e Biotecnologia. Universidade Federal do Amazonas; Grupo de Pesquisa Saúde e Desempenho Humano-GPeSDH/ UFAM-AM, Brasil.

\section{Grasiely Faccin Borges}

Docente Adjunta do Instituto de Saúde e Biotecnologia; Universidade Federal do Amazonas; Grupo de Pesquisa Saúde e Desempenho Humano-GPeSDH/ UFAM-AM, Brasil.

E-mail: grasiely.borges@gmail.com
RESUMO: O termo crioterapia é utilizado para descrever a aplicação de modalidades de frio que tem uma variação de temperatura de $0^{\circ} \mathrm{C}$ a $18,3^{\circ} \mathrm{C}$. Desse modo, o objetivo deste trabalho é apresentar e discutir os métodos de aplicação da crioterapia divulgados nas publicações científicas brasileiras nos últimos dez anos. Para a realização desta revisão sistemática da literatura, utilizou-se como fonte de pesquisa os artigos de periódicos científicos nacionais nas bases de dados Scientific Eletronic Library Online (Scielo), Literatura Latino-Americana e do Caribe em Ciências da Saúde (Lilacs) e Google Acadêmico. Os descritores na língua portuguesa utilizados na pesquisa foram: "crioterapia", "hipotermia induzida" e "resposta ao choque frio". Foram utilizados artigos publicados entre os anos de 2004 e 2014. Foram classificados 21 artigos de acordo com os critérios de inclusão e exclusão. $\mathrm{O}$ método de aplicação da crioterapia mais frequente foi a aplicação com bolsa de gelo ou compressa de gelo, que consiste em resfriamento que se concentra na pele em até $1 \mathrm{~cm}$ de profundidade, geralmente aplicado com um tempo estimado entre 15 a 30 minutos. Com a realização deste trabalho verificou-se que a crioterapia é um excelente recurso terapêutico, sendo utilizada de forma efetiva para provocar redução da sensibilidade, da dor, pode também ajudar na redução da resistência à movimentação passiva e espasticidade. Não se mostrou eficaz no aumento da força muscular, da flexibilidade e em tratamentos para redução da gordura corporal.

PALAVRAS-CHAVE: Crioterapia; Hipotermia Induzida; Resposta ao Choque Frio.

\section{CRYOTHERAPY: METHODS AND APPLICATIONS IN RESEARCH IN BRAZIL A REVIEW}

\footnotetext{
ABSTRACT: Cryotherapy is the application of coldness, ranging between $0^{\circ} \mathrm{C}$ and $18.3^{\circ} \mathrm{C}$. Current research discusses application methods of cryotherapy in Brazilian scientific publications during the last ten years. The systematic review of the literature comprised research in article retrieved from national scientific journals in databases Scientific Electronic Library Online (SciELO), Latin-American and Caribbean Literature in Health Sciences (Lilacs) and Academic Google. Descriptors were: "cryotherapy", "induced hypothermia" and "response to cold shock". Articles published between 2004 and 2014 were used, out of which 21 were classified according to inclusion-exclusion criteria. The most used method in cryotherapy is the application of an ice bag or cold compress, by which the skin is cooled at a depth of $1 \mathrm{~cm}$, applied between 15 and 30 minutes. Cryotherapy is an excellent therapeutic method used effectively to reduce sensitiveness and pain. It may also help in the decrease of resistance to passive movement and spasticity, but failed to be efficient in the increase of muscle force, flexibility and in treatments for the reduction of body fat.
} 
KEY WORDS: Cryotherapy; Induced Hypothermia; Response to the Cold Shock.

\section{INTRODUÇÃO}

A crioterapia, ou "terapia por frio", em outras palavras, é aplicação terapêutica de qualquer substância ao corpo que resulta em remoção de calor corporal, diminuindo, assim, a temperatura dos tecidos (FERREIRA; FERNANDES, 2012; BRANCO et al., 2005). O termo crioterapia é utilizado para descrever a aplicação de modalidades de frio que tem uma variação de temperatura de $0^{\circ} \mathrm{C}$ a $18,3^{\circ} \mathrm{C}$. Durante a crioterapia, o calor é retirado do corpo e absorvido pela modalidade de frio, fazendo com que o corpo responda com uma série de respostas locais e sistêmicas. A magnitude desses efeitos está relacionada com a temperatura da modalidade, a duração do tratamento, e a superfície exposta ao tratamento (STARKEY, 2001; FREITAS; LUZARDO, 2013).

Apesar das primeiras descrições da utilização terapêutica do frio (neve e gelo natural) remontar à antiga Grécia, só em 1850 surgiu a primeira máquina de gelo de uso hospitalar, utilizada nos Estados Unidos da América para diminuir a temperatura em doente com malária (BRANCO et al., 2005). Há relatos do uso desta técnica no século 2500 A.C., pelo povo egípcio, como método analgésico e anti-inflamatório. Estas características levaram à difusão da crioterapia durante o século XIX, pelo médico do exército de Napoleão Bonaparte, sendo esta técnica a mais utilizada em casos de amputação de membros. Durante a década de 1970, este método passou a ser utilizado como prática comum em casos de lesões desportivas, sendo que já havia registros médicos, científicos do hábito e instrução de banhos frios em 129 a 201 D.C. (KNIGHT, 2000).

Atualmente, esta forma terapêutica, em especial o gelo, é amplamente utilizada na medicina esportiva. Todavia, esta técnica não é utilizada apenas para o tratamento de lesões ou traumas, também é utilizada para tratamentos estéticos, como tratamento de gordura localizada (lipodistrofia) e flacidez, além de remoção de protuberâncias esteticamente indesejáveis (crio cirurgia, utilizando-se nitrogênio líquido), como por exemplo, verrugas assim como em outros tratamentos (CARVALHO; CHIERICHETTI, 2006; ANDREWS; HARRELSON; WILK, 2000; MACHADO et al., 2014).

Apesar de ser um tema muito discutido, ainda existem dúvidas sobre seu uso e também sua eficácia. $\mathrm{O}$ objetivo desta revisão sistemática foi apresentar e discutir os métodos de aplicação da crioterapia nas publicações científicas brasileiras nos últimos dez anos.

\section{MATERIAIS E MÉTODOS}

Para a realização desta revisão sistemática da literatura, utilizou-se como fonte de pesquisa os artigos de periódicos científicos nacionais, nas bases de dados Scientific Eletronic Library Online (Scielo), Literatura Latino-Americana e do Caribe em Ciências da Saúde (Lilacs) e Google Acadêmico.

Os descritores na língua portuguesa utilizados na pesquisa foram: "crioterapia", "hipotermia induzida" e "resposta ao choque frio". Foram utilizados artigos publicados entre os anos de 2004 e 2014, indexados na língua portuguesa. Como critério de inclusão, os artigos deveriam estar relacionados ao tema crioterapia e seus efeitos em humanos. Foram excluídas teses, dissertações, monografias, material informativo, os artigos que se referiam ao uso de crioterapia em animais, e materiais bibliográficos que não apresentavam disponibilidade de referência.

$\mathrm{Na}$ realização das buscas, com o descritor "crioterapia", foram encontrados no Google Acadêmico 6.280 artigos, desses foram selecionados 100, e classificado 01. No Scielo foram encontrados 97 artigos, desses foram selecionados 50 e classificados 02 de acordo com os critérios estabelecidos. No Lilacs foram encontrados 321 artigos e foram selecionados 03 de acordo com os critérios de inclusão e exclusão.

Com o descritor "hipotermia induzida", no Google Acadêmico foram encontrados 2.510 artigos, dos quais foram escolhidos 100 e selecionados 03 . No Scielo foram encontrados 17 artigos e selecionados apenas 02 de acordo com os critérios. No Lilacs foram encontrados 555 artigos, desses foram escolhidos 100 e selecionados 03 . 
Com a utilização do descritor "resposta ao choque frio", no Google Acadêmico foram encontrados 781 artigos, desses foram escolhidos 100 e selecionados 02. No Scielo foram encontrados 02 artigos, e os mesmos foram selecionados de acordo com os critérios de inclusão. No Lilacs foram encontrados 291 artigos, desses foram escolhidos 100 e selecionados 02 de acordo com os critérios desta revisão.

No total foram encontrados 10.854 artigos nas bases selecionadas, selecionados 716 e classificados 21 artigos, respeitando os critérios de inclusão e exclusão.

\section{RESULTADOS}

Foi encontrado um total de 10.854 artigos, desses foram selecionados um total de 716 dos quais foram classificados 21 artigos de acordo com os critérios de inclusão (Quadro 1).

No Quadro 01 foram apresentados os resultados dos estudos onde a aplicação da crioterapia não alcançou resultados esperados ou não foi eficaz. A crioterapia não foi eficaz na redução da lombalgia crônica, controle postural, aumento de força, aumento de flexibilidade, na redução de adiposidade corporal e foi contraindicada quando a sensibilidade precisa ser preservada.

Quadro 1. Estudos publicados em periódicos brasileiros onde a aplicação da crioterapia não foi eficaz ( $\mathrm{n}=09)$

(continua)

\begin{tabular}{|c|c|c|c|}
\hline Autor / Ano & $\begin{array}{l}\text { Amostra de } \\
\text { Estudo / Idade }\end{array}$ & $\begin{array}{l}\text { Método de Aplicação / Tempo de Aplicação } \\
\text { da Crioterapia }\end{array}$ & Conclusão \\
\hline $\begin{array}{l}\text { ABREU; } \text { SANTOS; } \\
\text { VENTURA, } 2011 .\end{array}$ & $\begin{array}{l}6 \text { mulheres, com } \\
\text { idade entre } 50 \text { e } 60 \\
\text { anos. }\end{array}$ & $\begin{array}{l}\text { A crioterapia foi feita com bolsa de gelo moído, } \\
\text { aplicação de } 20 \text { minutos durante } 5 \text { dias. }\end{array}$ & $\begin{array}{l}\text { A terapia combinada não apresentou } \\
\text { nenhuma melhora significativa na } \\
\text { lombalgia crônica. }\end{array}$ \\
\hline $\begin{array}{l}\text { CARVALHO et al., } \\
2012 .\end{array}$ & $\begin{array}{l}20 \text { pessoas de } \\
\text { ambos os sexos, } \\
\text { com idade entre } 20 \\
\text { a } 27 \text { anos. }\end{array}$ & $\begin{array}{l}\text { Bolsa de gelo flexível, tamanho médio, contendo } \\
0,6 \mathrm{~kg} \text {. Cada bolsa de gel permaneceu por no } \\
\text { mínimo oito horas no congelador antes de ser } \\
\text { utilizada. Aplicação: } 25 \text { minutos. }\end{array}$ & $\begin{array}{l}\text { A associação da crioterapia com } \\
\text { qualquer outra terapia que necessite } \\
\text { da sensibilidade preservada é } \\
\text { contraindicada. }\end{array}$ \\
\hline $\begin{array}{l}\text { CASSOLATO et al., } \\
2012 .\end{array}$ & $\begin{array}{l}9 \text { indivíduos, de } \\
\text { ambos os sexos, } \\
\text { com idade de } 21 \\
\text { anos. }\end{array}$ & $\begin{array}{l}\text { Imersão dos tornozelos dominantes à altura de } \\
3 \mathrm{~cm} \text { acima do maléolo lateral, por } 15 \text { minutos, } \\
\text { em mistura de água e gelo a } 5^{\circ} \mathrm{C} \text {. Além disso, } \\
\text { bacias com diâmetro de } 80 \mathrm{~cm} \text { e } 25 \mathrm{~cm} \text { de } \\
\text { altura comportaram a mistura de água e gelo, } \\
\text { para posterior imersão dos tornozelos por } 15 \\
\text { minutos. }\end{array}$ & $\begin{array}{l}\text { A crioterapia não influenciou no } \\
\text { controle postural, em condição de } \\
\text { postura ereta estática, de indivíduos } \\
\text { saudáveis, pela avaliação do centro de } \\
\text { pressão. }\end{array}$ \\
\hline CORRÊA et al., 2012. & $\begin{array}{l}13 \text { mulheres com } \\
\text { idade entre } 18 \text { a } 30 \\
\text { anos. }\end{array}$ & $\begin{array}{l}\text { Compressa de gelo ( } 500 \mathrm{~g} \text { de gelo triturado } \\
\text { contido em um saco plástico), cobrindo } \\
\text { totalmente o músculo quadríceps, sendo fixada } \\
\text { por meio de uma bandagem elástica por } 30 \\
\text { minutos. }\end{array}$ & $\begin{array}{l}\text { Não houve aumento da força } \\
\text { isométrica do quadríceps por até } 60 \\
\text { minutos após aplicação. }\end{array}$ \\
\hline FARIAS et al., 2010. & $\begin{array}{l}13 \text { voluntárias } \\
\text { do sexo feminino } \\
\text { com idades que } \\
\text { variaram entre 16- } \\
20 \text { anos. }\end{array}$ & $\begin{array}{l}\text { Aplicação de panqueca fria durante } 20 \text { minutos } \\
\text { na região da prega cutânea tricipital. O gelo foi } \\
\text { triturado e colocado em uma toalha úmida. }\end{array}$ & $\begin{array}{l}\text { Não foi observado superioridade da } \\
\text { Criotens em relação à Crioterapia e a } \\
\text { TENS. }\end{array}$ \\
\hline $\begin{array}{l}\text { MACHADO et al., } \\
2014 .\end{array}$ & $\begin{array}{l}33 \text { mulheres na } \\
\text { faixa etária entre } 18 \\
\text { e } 29 \text { anos. }\end{array}$ & $\begin{array}{l}\text { Três grupos: aplicação de gel crioterápico sem } \\
\text { bandagem com bandagem por } 30 \text { minutos. }\end{array}$ & $\begin{array}{l}\text { Não alterou a adiposidade corporal } \\
\text { das mulheres. }\end{array}$ \\
\hline $\begin{array}{l}\text { MORTARI; MÂNICA; } \\
\text { PIMENTEL, } 2009 .\end{array}$ & $\begin{array}{l}18 \text { mulheres, com } \\
\text { idade entre } 18 \text { e } 24 \\
\text { anos. }\end{array}$ & $\begin{array}{l}\text { Gelo picado em sacos plásticos sob e sobre } \\
\text { ambas as coxas. Aplicação: } 15 \text { minutos. }\end{array}$ & $\begin{array}{l}\text { A técnica mantém-relaxa da FNP gerou } \\
\text { maiores valores no pico de torque em } \\
\text { relação à crioterapia. }\end{array}$ \\
\hline
\end{tabular}


(conclusão)

\begin{tabular}{|l|l|l|l|}
\hline $\begin{array}{l}\text { SILVA; IMOTO; } \\
\text { CROCI, 2007. }\end{array}$ & $\begin{array}{l}25 \text { indivíduos, de } \\
\text { ambos os sexos, } \\
\text { com idade de 58 a } \\
78 \text { anos. }\end{array}$ & Aplicação de gelo por 20 minutos. & $\begin{array}{l}\text { Não houve relação de ganho de } \\
\text { amplitude, flexibilidade e força } \\
\text { associado à termoterapia. }\end{array}$ \\
\hline SILVA et al., 2010. & $\begin{array}{l}40 \quad \text { jovens } \\
\text { voluntários, de } \\
\text { ambos os sexos, } \\
\text { com idade de 21 } \\
\text { anos. }\end{array}$ & $\begin{array}{l}\text { Uma bolsa de gel resfriada sobre a região } \\
\text { posterior da coxa. Foi aplicada compressão } \\
\text { na região por meio de bandagem elástica e } \\
\text { foi coberta por uma bandagem para evitar } \\
\text { ulcerações provocadas pelo frio. Aplicação: } 20 \\
\text { minutos. }\end{array}$ & $\begin{array}{l}\text { O alongamento estático proporcionou } \\
\text { aumento da flexibilidade de músculos } \\
\text { encurtados independentemente do } \\
\text { uso de recursos térmicos. }\end{array}$ \\
\hline
\end{tabular}

FNP = Facilitação neuromuscular proprioceptiva; TENS = Estimulação elétrica transcutânea do nervo.

No Quadro 2 são apresentados os resultados onde o uso da crioterapia foi eficaz, especificamente na redução da temperatura tecidual, na modificação da atividade eletromiográfica, na redução da dor, no aumento da amplitude articular, nas alterações metabólicas tanto em indivíduos saudáveis quanto em diabéticos, na redução do escape salivar.

Quadro 2. Estudos selecionados sobre a utilização da crioterapia em periódicos brasileiros que apresentaram resultados eficazes $(\mathrm{n}=12)$

(continua)

\begin{tabular}{|c|c|c|c|}
\hline Autor / Ano & $\begin{array}{l}\text { Amostra de Estudo } \\
\text { / Idade }\end{array}$ & $\begin{array}{l}\text { Método de Aplicação / Tempo de } \\
\text { Aplicação da Crioterapia }\end{array}$ & Conclusão \\
\hline $\begin{array}{l}\text { BRASILEIRO; FARIA; } \\
\text { QUEIROZ, } 2007 .\end{array}$ & $\begin{array}{l}40 \text { sujeitos de ambos } \\
\text { os sexos, com idade } \\
\text { média de } 21 \text { anos. }\end{array}$ & $\begin{array}{l}\text { Aplicação de compressas de gelo de } 1,5 \mathrm{~kg} \text {, } \\
\text { sob a forma de pacotes, na face posterior da } \\
\text { coxa por } 15 \text { minutos. }\end{array}$ & $\begin{array}{l}\text { Efeitos agudos foram maiores com } \\
\text { resfriamento na flexibilidade dos } \\
\text { músculos isquiotibiais. }\end{array}$ \\
\hline $\begin{array}{l}\text { CARVALHO; } \\
\text { CHIERICHETTI, } 2006 .\end{array}$ & $\begin{array}{l}18 \text { indivíduos, com } \\
\text { idade entre } 18 \text { e } 25 \\
\text { anos, de ambos os } \\
\text { sexos. }\end{array}$ & $\begin{array}{l}\text { Crioterapia com gelo na coxa esquerda } \\
\text { e mistura de gelo e água na coxa direita, } \\
\text { na região do quadríceps femoral por } 15 \\
\text { minutos. }\end{array}$ & $\begin{array}{l}\text { Ambas as modalidades promoveram } \\
\text { redução da temperatura. }\end{array}$ \\
\hline $\begin{array}{l}\text { COELHO; } \\
\text { PEREIRA; PEREIRA, } \\
2008 .\end{array}$ & $\begin{array}{l}14 \text { indivíduos do } \\
\text { gênero masculino. } \\
\text { Idades entre } 18 \text { e } 24 \\
\text { anos. }\end{array}$ & $\begin{array}{l}\text { Aplicação de } 2 \text { bolsas plásticas com cubos } \\
\text { de gelo em quantidade suficiente para } \\
\text { envolver toda a superfície do tornozelo por } \\
20 \text { minutos. }\end{array}$ & $\begin{array}{l}\text { A crioterapia influencia a atividade } \\
\text { eletromiográfica dos músculos tibial } \\
\text { anterior e fibular longo. }\end{array}$ \\
\hline CORREIA et al., 2010. & $\begin{array}{l}7 \text { voluntários de } \\
\text { ambos os sexos com } \\
\text { idade média de } 67 \\
\text { anos. }\end{array}$ & $\begin{array}{l}\text { Aplicação de gelo na musculatura extensora } \\
\text { do punho e dedos (crioestimulação) durante } \\
1 \text { minuto e } 40 \text { segundos. }\end{array}$ & $\begin{array}{l}\text { A aplicação local, contínua e rápida da } \\
\text { crioterapia, associada à cinesioterapia, } \\
\text { parece ser eficiente. }\end{array}$ \\
\hline DAMBROS et al., 2012. & $\begin{array}{l}\text { Indivíduos, ambos os } \\
\text { gêneros acima de } 18 \\
\text { anos. }\end{array}$ & $\begin{array}{l}\text { Aplicação de compressa com gelo (realizada } \\
\text { utilizando-se gelo picado envolto em } \\
\text { material plástico estéril) na região anterior } \\
\text { do joelho acometido, com o membro em } \\
\text { elevação, por } 20 \text { minutos. }\end{array}$ & $\begin{array}{l}\text { A crioterapia no pós-operatório } \\
\text { imediato, associada a um protocolo de } \\
\text { exercícios, foi efetiva para melhora da } \\
\text { dor e ADM do joelho. }\end{array}$ \\
\hline SILVA et al., 2012. & $\begin{array}{l}2 \text { voluntárias do sexo } \\
\text { feminino, com idades } \\
\text { de } 20 \text { e } 25 \text { anos. }\end{array}$ & $\begin{array}{l}\text { Gel crioterápico, envolvido em bandagens } \\
\text { umedecidas com água, abrangendo mais de } \\
70 \% \text { da área corporal (membros superiores, } \\
\text { abdômen, glúteo e membros inferiores, } \\
\text { evitando as áreas de articulações) por } 20 \\
\text { minutos. }\end{array}$ & $\begin{array}{l}\text { O aumento metabólico promovido } \\
\text { pela crioterapia, em grande área } \\
\text { corporal, parece ser capaz de interferir } \\
\text { no equilíbrio térmico-sistêmico e } \\
\text { ativar os mecanismos de controle da } \\
\text { temperatura central. }\end{array}$ \\
\hline $\begin{array}{l}\text { FERREIRA; } \\
\text { FERNANDES, } 2012 .\end{array}$ & $\begin{array}{l}\text { Uma paciente com } 22 \\
\text { anos de idade. }\end{array}$ & $\begin{array}{l}\text { Aplicação de gelo, sob a forma de pacote, } \\
\text { no ventre do músculo bíceps braquial do } \\
\text { membro superior esquerdo por } 30 \text { minutos. }\end{array}$ & $\begin{array}{l}\text { Foi eficaz na diminuição da } \\
\text { espasticidade } \\
\text { consequente ganho na ADM articular. }\end{array}$ \\
\hline
\end{tabular}


(conclusão)

\begin{tabular}{|c|c|c|c|}
\hline $\begin{array}{l}\text { FRANCISCO et al., } \\
2013 .\end{array}$ & $\begin{array}{l}114 \text { puérperas de } 18 \text { a } \\
38 \text { anos. }\end{array}$ & $\begin{array}{l}\text { Três grupos usaram bolsa de gelo. A } \\
\text { aplicação durou } 10,15 \text { ou } 20 \text { minutos. }\end{array}$ & $\begin{array}{l}\text { Dez minutos de aplicação foram } \\
\text { suficientes para reduzir a temperatura } \\
\text { perineal. }\end{array}$ \\
\hline $\begin{array}{l}\text { NUNES; VARGENS, } \\
2007 .\end{array}$ & 21 parturientes. & $\begin{array}{l}\text { Compressa de gelo em cinta apropriada } \\
\text { e aplicada sobre a região lombar com a } \\
\text { parturiente em decúbito lateral esquerdo. } \\
\text { Foram feitas até duas aplicações em cada } \\
\text { parturiente, na fase ativa do período de } \\
\text { dilatação, quando o colo do útero alcançou } \\
7 \text { e } 9 \mathrm{~cm} \text { por } 20 \text { minutos. }\end{array}$ & $\begin{array}{l}\text { A crioterapia pode ser aplicada para } \\
\text { alívio da dor no parto. }\end{array}$ \\
\hline ROSA et al., 2005. & $\begin{array}{l}\text { Criança de } 3 \text { anos e } \\
08 \text { meses de idade, do } \\
\text { gênero masculino. }\end{array}$ & $\begin{array}{l}\text { Doze sessões de } 10 \text { minutos, todos os dias da } \\
\text { semana, uma vez ao dia, durante dois meses. } \\
\text { O gelo foi passado primeiramente na região } \\
\text { perioral com movimento circular, uma vez } \\
\text { no sentido horário e outra no anti-horário, } \\
\text { de forma que atingisse toda a extensão do } \\
\text { músculo orbicular da boca. }\end{array}$ & $\begin{array}{l}\text { A crioterapia mostrou-se eficaz } \\
\text { na redução do escape de saliva } \\
\text { favorecendo as funções oromotoras e } \\
\text { a alimentação da criança. }\end{array}$ \\
\hline SILVA et al., 2012. & $\begin{array}{l}15 \text { pacientes, de } \\
\text { ambos os sexos, com } \\
\text { idade média de } 56 \\
\text { anos. }\end{array}$ & $\begin{array}{l}\text { Aplicação de gelo na região anterior e medial } \\
\text { do braço do hemicorpo acometido por meio } \\
\text { de sacos plásticos com gelo, utilizando um } \\
\text { pano de limpeza entre a pele e o saco de } \\
\text { gelo e uma atadura de crepom para fixá-lo } \\
\text { ao braço, por um período de } 20 \text { minutos. }\end{array}$ & $\begin{array}{l}\text { Houve uma redução da resistência à } \\
\text { movimentação passiva dos flexores e } \\
\text { extensores de cotovelo. }\end{array}$ \\
\hline SUZANO; SÁ, 2010. & $\begin{array}{l}9 \text { indivíduos de ambos } \\
\text { os sexos com idade de } \\
18 \text { a } 60 \text { anos. }\end{array}$ & $\begin{array}{l}\text { Um protocolo de agente crioterapêutico: } \\
\text { imersão em água + gelo, bolsa termogel ou } \\
\text { saco de gelo moído por } 20 \text { minutos. }\end{array}$ & $\begin{array}{l}\text { A imersão em água gelada nos } \\
\text { diabéticos foi o recurso que se } \\
\text { comportou com padrão mais } \\
\text { fisiológico quando comparado aos } \\
\text { saudáveis. }\end{array}$ \\
\hline
\end{tabular}

$\mathrm{ADM}=$ Amplitude de movimento; FNP = Facilitação neuromuscular proprioceptiva.

O método de aplicação da crioterapia que mais se destacou foi a aplicação com bolsa de gelo ou compressa de gelo $(73,91 \%)$, que consiste em resfriamento que se concentra na pele em até $1 \mathrm{~cm}$ de profundidade. Outros métodos como bolsa de gel (13,04\%), crioimersão ou crioestimulação (8,70\%), também foram utilizados nos estudos classificados desta revisão gel canforado e panqueca fria $(4,35 \%)$.

Nos estudos selecionados, o tempo de aplicação variou de 10 a 30 minutos, onde a maioria, 47,62\%, dos artigos aplicaram durante 20 minutos, seguido por 15 minutos em 19,05\% dos estudos, 30 minutos em 14,29\%, 25 minutos em $9,52 \%$ e 10 minutos em $9,52 \%$ dos estudos classificados, sendo que uma variação ocorreu de acordo com a patologia, local de aplicação e características individuais como por exemplo o percentual de gordura corporal.

\section{DISCUSSÃO}

Os efeitos locais da aplicação da crioterapia incluem vasoconstrição com redução do fluxo sanguíneo e diminuição da taxa metabólica, redução de resíduos celulares, redução da inflamação, redução da dor, redução do espasmo muscular. Observa-se também que os efeitos são diminuição de edema e metabolismo, hiperemia no local da aplicação, diminuição do processo inflamatório e regeneração tecidual (STARKEY, 2001; FREITAS; LUZARDO, 2013). O frio pode ser aplicado quando se pretende analgesia, causada por aumentar a tolerância à dor por diminuir a velocidade da condução nervosa e a produção dos mediadores de dor com redução em sua percepção, redução da inflamação e do edema na fase aguda de traumatismos ou queimaduras, redução de metabolismo celular, redução da hemorragia, diminuição da espasticidade, facilitação de técnicas cinesiológicas, normalização térmica em situações de hiperaquecimento 
muscular e estimulação de regeneração e do trofismo celular em patologias traumáticas crônicas, promove restauração estrutural e favorece o processo de reabilitação (BRANCO et al., 2005; MACEDO; GUIRRO, 2013).

Para obter os benefícios terapêuticos, a temperatura da pele deve cair para aproximadamente $13,80{ }^{\circ} \mathrm{C}$ para que ocorra a redução local do fluxo sanguíneo, e para cerca de $14,40{ }^{\circ} \mathrm{C}$ para que ocorra a analgesia. A duração dos tratamentos com frio depende da profundidade do tecido alvo e do objetivo do tratamento. Tecidos mais profundos devem ser esfriados por períodos mais longos do que os superficiais. Fontes muito frias devem ser usadas por períodos mais curtos do que as fontes que são meramente frias (HAYES, 2002).

A escolha correta do método de aplicação deve ser baseada, principalmente, de acordo com a área a ser tratada, sendo que o tempo de aplicação varia tanto em relação ao método utilizado como a área, isto é, uma articulação que apresenta menor espessura do tecido adiposo, o tempo necessário para atingir o resfriamento é menor do que em uma outra área que possui uma maior quantidade de tecido adiposo (BRANCO et al., 2005).

A aplicação pode ser realizada de forma dinâmica ou estática. A aplicação estática da crioterapia é utilizada quando se pretende efeitos vasoconstritores e antiálgicos, por exemplo, na fase aguda de um traumatismo. A temperatura cutânea a atingir é de $15^{\circ} \mathrm{C}$ e o período de aplicação deverá ser repetido várias vezes, não excedendo os 20 minutos de cada vez. Este efeito pode ser também obtido com imersão em água fria $\left(4-10{ }^{\circ} \mathrm{C}\right)$ durante 10 a 20 minutos. Com esta técnica, o arrefecimento dos tecidos profundos é mais rápido e mais duradouro. No entanto alguns autores defendem aplicação de frio superior a 20 minutos, considerando que este período é insuficiente para o arrefecimento dos tecidos mais profundos (BRANCO et al., 2005; FREITAS; LUZARDO, 2013).

A aplicação dinâmica consiste em uma massagem circular rápida por períodos de 2 a 3 segundos, durante 7 a 10 minutos e é utilizada quando se pretende obter hiperemia reativa e uma analgesia menos pronunciada, como por exemplo, na fase crônica (mais de 8 dias) de patologia traumática (BRANCO et al., 2005).
Durante a aplicação de crioterapia, observa-se uma primeira fase de sensação inicial de frio. A segunda fase é de dor e desconforto, e a terceira de analgesia ou anestesia. A quarta fase produz vasodilatação reflexa ou paralítica profunda. Essas fases duram aproximadamente três minutos cada, dependendo da modalidade utilizada (CARVALHO et al., 2012).

A crioterapia pode ser aplicada de diversas maneiras: compressas geladas, por meio de recipientes, toalhas ou saco plástico com gelo picado, compressas de gel, compressas químicas, imersão em água e gelo, gelo seco, spray químico (DENEGAR, 2003; MACEDO; GUIRRO, 2013).

1. Pacote de gelo - Após 20 minutos de aplicação do pacote de gelo, o resfriamento se concentra na pele e em até $1 \mathrm{~cm}$ de profundidade, o que pode ser associada à diminuição da percepção da dor pela grande concentração de receptores nos tecidos superficiais, comprovando a analgesia (MACEDO; GUIRRO, 2013). O tempo de aplicação do pacote de gelo é estimado entre 15 a 30 minutos para analgesia, diminuição dos espasmos musculares e controle de edema (MACEDO; GUIRRO, 2013; FERREIRA; FERNANDES, 2012).

2. Crioimersão - A imersão em água fria desencadeia a diminuição da temperatura da pele e dos tecidos superficiais após 5 minutos de utilização. $\mathrm{Na}$ prática clínica ocorrem divergências em relação ao tempo de aplicação e a temperatura de água. Estudos realizaram imersão em temperatura (MACEDO; GUIRRO, 2013).

3. Massagem com gelo - Utilizada por profissionais do esporte e atletas com objetivo de devolver o melhor desempenho em um menor tempo. Não deve ser utilizada como socorro de urgência ou na resposta inflamatória aguda, pois não pode ser associada à compressão. Porém reduz a dor antes de exercícios terapêuticos e o desconforto pós-exercício. Também pode ser utilizada para dessensibilizar pontos gatilhos na dor miofacial (DENEGAR, 2003). O gelo deve ser aplicado diretamente na pele com 
movimentos de deslizamentos circulares. $\mathrm{O}$ tempo de aplicação deve estar entre 7 e 10 minutos.

4. Bolsa de gel - $\mathrm{O}$ uso de bolsa de gel resfriada ou congelada é frequente pela sua fácil aplicação, principalmente entre leigos e atletas. Sua evidência não é comprovada, são poucos os estudos apresentados com boa qualidade, o que dificulta a comparação e a discussão dos resultados (MACEDO; GUIRRO, 2013).

5. Criospray-Resultamem resfriamentosuperficial e rápido em função de sua evaporização. Reduz a analgesia e o espasmo muscular. Porém não reduz a hemorragia, pois não tem efeito de diminuição da temperatura de tecidos profundos. A utilização da crioterapia à base de gel canforado em mentol é antiga e largamente utilizada pelos fisioterapeutas para o tratamento de adiposidades localizadas de forma conservadora. $\mathrm{O}$ mecanismo de ação da crioterapia a base de gel canforado e mentol se dá pelo resfriamento da pele por meio da evaporização dos componentes do gel (MACHADO et al., 2012).

Sobre os resultados dos trabalhos estudados, verificou-se que a crioterapia associada à cinesioterapia, ao calor ultrassônico, ao calor superficial (infravermelho), e à estimulação elétrica neuromuscular, se mostrou eficaz na redução da resistência à movimentação passiva, e melhora da amplitude de movimento (DAMBROS; MARTIMBIANCO; POLACHINI, 2012) de indivíduos espásticos acometidos por paralisia cerebral (CORREIA et al., 2010; FEREIRA; FERNANDES, 2012; CRUZ; GOTARDO; JORGE, 2006; SILVA et al., 2012).

A aplicação de crioterapia na força isométrica do quadríceps, na força muscular dos flexores e extensores de joelho e na flexibilidade dos músculos isquiotibiais não se mostrou eficaz, pois não houve alterações significativas após sua aplicação (MORTARI; MÂNICA; PIMENTEL, 2009; SILVA et al., 2010; CORRÊA; PELEGRINI; AMBROSIO, 2012; BRASILEIRO; FARIA; QUEIROZ, 2007).

A eficácia analgésica da eletroestimulação nervosa transcutânea (TENS), e a crioterapia na lombalgia crônica, não comprovou melhora significativa em relação às modalidades separadas, porém as modalidades analgésicas, associação de crioetrapia e TENS foram eficazes em aliviar a dor lombar crônica (ABREU; SANTOS; VENTURA, 2011).

\section{CONCLUSÃO}

Com a realização deste trabalho, verificouse entre as publicações brasileiras, que a crioterapia é um excelente recurso terapêutico, sendo utilizada de forma efetiva para provocar redução da sensibilidade, sendo contraindicada quando essa deve ser preservada, diminui também a dor em diversos aspectos, incluído o parto, pode também ajudar na redução da resistência à movimentação passiva e espasticidade. Não se mostrou eficaz no aumento da força muscular, da flexibilidade, e nem em tratamentos para redução da gordura corporal.

Verificou-se que mais estudos são necessários sobre a utilização deste recurso terapêutico, tanto sobre o tempo de aplicação, quanto sobre as técnicas que devem ser utilizadas em cada caso específico, faltam evidências na literatura científica sobre a utilização da crioterapia propriamente.

\section{REFERÊNCIAS}

ABREU, E. A.; SANTOS, J. D. M.; VENTURA, P. L. Eficácia analgésica da associação da eletroestimulação nervosa transcutânea e crioterapia na lombalgia crônica, Revista Dor, São Paulo, v. 12, n. 1, p. 23-8, jan./mar. 2011.

ANDREWS, J. R.; HARRELSON, G. L.; WILK, K. E. Reabilitação física das lesões desportivas. 2. ed. Rio de Janeiro: Guanabara Koogan, 2000.

BRANCO, P. S. et al. Tema de reabilitação agentes físicos: crioterapia, termoterapia, diatermia. Porto, Portugal: [s.n.], 2005. p. 11-20.

BRASILEIRO, J. S.; FARIA, A. F. E.; QUEIROZ, L. L. Influência do resfriamento e do aquecimento local na flexibilidade dos músculos isquiostibiais. Rev Bras Fisioter., São Carlos, v. 11, n. 1, p. 57-61, jan./fev. 2007. 
CARVALHO, G. A.; CHIERICHETTI, H. S. L. Avaliação da sensibilidade cutânea palmar nas aplicações de crioterapia por bolsa de gelo e bolsa de gel. R Bras Ci e Mov., v. 14, n. 1, p. 23-30, 2006.

CARVALHO, R. A.; MEDEIROS, D. L.; SOUZA, F. T.; PAULA, G. F.; BARBOSA, P. M.; VASCONCELLOS, P. R. O.; BUZANELLO, M. R.; BERTOLINI, G. R. F. Variação de temperatura do músculo quadríceps femoral exposto a duas modalidades de crioterapia por meio de termografia. Rev Bras Med Esporte, v. 18, n. 2, p. 109-11; mar./abr. 2012.

CASSOLATO, K. M.; ARTIFON, E. L.; BONFIM, A. E. O.; SCALCO, J.C.; NAVARRO, G.T.; CARVALHO, A.R.; BERTOLINI, G.R.F. Influência da crioterapia no controle postural da postura ereta em indivíduos saudáveis. Rev Bras Clin Med., São Paulo, v. 10, n. 5, p. 402-406, set./ out. 2012.

COELHO, M. V. C.; PEREIRA, L. G.; PEREIRA, R. Crioterapia no tornozelo e atividade eletromiográfica do tibial anterior e fibular durante o apoio unipodálico no balancinho. Perspectivas online, v. 2, n. 7, p. 98-101, 2008.

CORRÊA, J. B.; PELEGRINI, S.; AMBROSIO, R. P.; AFINI, L. F.; MINECHELLI, L. F.; LIEBANO, R. E. Efeitos da crioterapia no momento máximo de força isometria do quadríceps. R Bras Ci e Mov., v. 20, n. 4, p. 99-105, 2012.

CORREIA, A. C. S.; SILVA, J. D. S.; SILVA, L. V. C.; OLIVEIRA, D. A.; CABRAL, E. D. Crioterapia e cinesioterapia no membro superior espástico no acidente vascular cerebral: relato de caso. Fisioter. Mov., v. 23, n. 4, p. 555-63, out./ dez. 2010.

CRUZ, C. L.; GOTARDO, C. F. O.; JORGE, S. Influência da crioterapia e do calor superficial na espasticidade: relato de caso. Arq Ciênc Saúde Unipar, v. 7, n. 3, p. 253-257, 2003.

DAMBROS, C.; MARTIMBIANCO, A. L. C.; POLACHINI, L. O.; LAHOZ, G. L.; CHAMLIAN, T. R.; COHEN, M. Efetividade da crioterapia pós reconstrução do ligamento cruzado anterior. Acta Ortop bras., São Paulo, v. 20, n. 5, p. 285-290, 2012.
DENEGAR, C. R. Modalidades terapêuticas para lesões atléticas. Barueri: Manole, 2003.

FARIAS, R. S.; MELO, R. S.; MACHADO, Y. F.; LIMA, F. M.; ANDRADE, P. R. O uso da tens, crioterapia e criotens na resolução da dor. Rev Bras Ci Saúde, v. 14, n. 1, p. 27 36, 2010.

FERREIRA, A. A. S.; FERNANDES, D. S. S. L. Influência da Crioterapia e do calor ultrassônico na paralisia cerebral: relato de caso. Rev Neurocienc, v. 20, n. 4, p. 552-9, 2012.

FRANCISCO, A. A.; OLIVEIRA, S. M. J. V.; LEVENTHAL, L. C.; BOSCO, C. S. Crioterapia no pós-parto: tempo de aplicação e mudanças na temperatura perineal. Rev Esc Enferm USP, v. 47, n. 3, p. 555-61, 2013.

FREITAS, C.; LUZARDO, R. Crioterapia: efeitos sobre as lesões musculares. Rev Epist Transversalis, v. 4, n. 1, p. 1-7, 2013.

HAYES, K. W. Manual de agentes físicos: recursos fisioterapêuticos. 5. ed. Porto Alegre: Artmed, 2002.

KNIGHT, K. L. Crioterapia no tratamento das lesões esportivas. São Paulo: Manole, 2000.

MACEDO, C. S. G.; GUIRRO, R. R. J. Crioterapia: teoria e prática baseada em evidência. Profisio esportiva e traumato-ortópedica, Porto Alegre, ciclo 3, v. 1, p. 6595, 2013.

MACHADO, A. F. P.; SCHWARTZ, J.; RIBEIRO, S. M. L.; TACANI, P. M.; TACANI, R. E.; LIEBANO, R. E. Crioterapia com e sem bandagem na adiposidade localizada de mulheres jovens. ConScientiae Saúde, v. 11, n. 3, p. 420-428, 2014.

MORTARI, D. M.; MÂNICA, A. P.; PIMENTEL, G. L. Efeitos da crioterapia e facilitação neuromuscular proprioceptiva sobre a força muscular nas musculaturas flexora e extensora do joelho. Fisioter Pesqui., v. 16, n. 4, p. 1-5, 2009.

NUNES, S.; VARGENS, O. M. Acrioterapia como estratégia para alívio da dor no trabalho de parto: um estudo exploratório. R Enferm UERJ, v. 15, n. 3, p. 337, Rio de Janeiro, jul./set. 2007. 
ROSA, C. M. R.; LOPES, A. R.; SANTOS, F. F.; MOTTA, A. R. A crioterapia como recurso para diminuir a sialorréia em criança com disfunção neuromotora: relato de caso. Revista CEFAC, v. 7, n. 3, p. 300-306, jul./set. 2005.

SILVA, A. L. P.; IMOTO, D. M.; CROCI, A. T. Estudo comparativo entre a aplicação de crioterapia, cinesioterapia e ondas curtas no tratamento da osteoartrite de joelho. Acta Ortop Bras., v. 15, n. 4, p. 204-209, 2007.

SILVA, D. D.; BORGES, A. C. L.; LIMA, M. O.; LIMA, F. P. S.; FREITAS, S. T. T.; NOGUEIRA, D. V.; LUCARELI, P. R. G.; PAULA JUNIOR, A. R.; COGO, J. C. Resistência ao movimento e atividade eletromiográfica dos músculos flexores e extensores de cotovelo em pacientes hemiparéticos espásticos submetidos à crioterapia e estimulação elétrica neuromuscular. Rev Bras Eng Bioméd., v. 28, n. 3, p. 248-260, set. 2012.

SILVA, T. M.; DATSCH, A. L.; FRANZIN, R. C.; ANGELI, S.; COSTA, P. H. Uso da crioterapia como ferramenta para o aumento de consumo de $\mathrm{O}_{2}$ no tratamento da adiposidade localizada: relato de caso. Perspectivas Online: biológicas e saúde, Campos dos Gouytazes, v. 5, n. 2, p. 31-38, 2012.

SILVA, S. A; OLIVEIRA, D. J.; JAQUES, M. J. N.; ARAUJO, R. C. Efeito da crioterapia e termoterapia associados ao alongamento estático na flexibilidade dos músculos isquiotibiais. Motricidade. v. 6, n. 4, p. 55-62, 2010.

STARKEY, C. Recursos terapêuticos em fisioterapia. 2. ed. Barueri: Manole, 2001.

SUZANO, P. R.; SÁ, B. V. W. Variação da temperatura superficial da pele utilizando três recursos da crioterapia em indivíduos diabéticos. Rev Eletr Novo Enfoque, v. 10, n. 10, p. 9-17, 2010. 